\title{
ORDER SYSTEMS, IDEALS AND RIGHT FIXED MAPS OF SUBTRACTION ALGEBRAS
}

\author{
Young Bae Jun, Chul Hwan Park, and Eun Hwan Roh
}

\begin{abstract}
Conditions for an ideal to be irreducible are provided. The notion of an order system in a subtraction algebra is introduced, and related properties are investigated. Relations between ideals and order systems are given. The concept of a fixed map in a subtraction algebra is discussed, and related properties are investigated.
\end{abstract}

\section{Introduction}

B. M. Schein [14] considered systems of the form $(\Phi ; \circ, \backslash)$, where $\Phi$ is a set of functions closed under the composition "o" of functions (and hence $(\Phi ; \circ)$ is a function semigroup) and the set theoretic subtraction "\" (and hence $(\Phi ; \backslash)$ is a subtraction algebra in the sense of [2]). He proved that every subtraction semigroup is isomorphic to a difference semigroup of invertible functions. B. Zelinka [15] discussed a problem proposed by B. M. Schein concerning the structure of multiplication in a subtraction semigroup. He solved the problem for subtraction algebras of a special type, called the atomic subtraction algebras. Y. B. Jun et al. [10] introduced the notion of ideals in subtraction algebras and discussed characterization of ideals. In [6], Y. B. Jun and H. S. Kim established the ideal generated by a set, and discussed related results. Y. B. Jun and K. H. Kim [11] introduced the notion of prime and irreducible ideals of a subtraction algebra, and gave a characterization of a prime ideal. They also provided a condition for an ideal to be a prime/irreducible ideal. In this paper, we give conditions for an ideal to be irreducible. We introduce the notion of an order system in a subtraction algebra, and investigate related properties. We provide relations between ideals and order systems. We deal with the concept of a fixed map in a subtraction algebra, and investigate related properties.

\section{Preliminaries}

By a subtraction algebra we mean an algebra $(X ;-)$ with a single binary operation "-" that satisfies the following identities: for any $x, y, z \in X$,

Received January 27, 2007; Revised November 27, 2007.

2000 Mathematics Subject Classification. 03G25, 06B10, 06D99.

Key words and phrases. (weak, complicated) subtraction algebra, (irreducible) ideal, order system, right fixed map, kernel. 
(S1) $x-(y-x)=x$;

(S2) $x-(x-y)=y-(y-x)$;

(S3) $(x-y)-z=(x-z)-y$.

The last identity permits us to omit parentheses in expressions of the form $(x-y)-z$. The subtraction determines an order relation on $X: a \leq b \Leftrightarrow$ $a-b=0$, where $0=a-a$ is an element that does not depend on the choice of $a \in X$. The ordered set $(X ; \leq)$ is a semi-Boolean algebra in the sense of [2], that is, it is a meet semilattice with zero 0 in which every interval $[0, a]$ is a Boolean algebra with respect to the induced order. Here $a \wedge b=a-(a-b)$; the complement of an element $b \in[0, a]$ is $a-b$; and if $b, c \in[0, a]$, then

$$
\begin{aligned}
b \vee c & =\left(b^{\prime} \wedge c^{\prime}\right)^{\prime}=a-((a-b) \wedge(a-c)) \\
& =a-((a-b)-((a-b)-(a-c))) .
\end{aligned}
$$

In a subtraction algebra, the following are true (see $[10,11])$ :
(a1) $(x-y)-y=x-y$.
(a2) $x-0=x$ and $0-x=0$.
(a3) $(x-y)-x=0$.
(a4) $x-(x-y) \leq y$.
(a5) $(x-y)-(y-x)=x-y$.
(a6) $x-(x-(x-y))=x-y$.
(a7) $(x-y)-(z-y) \leq x-z$.
(a8) $x \leq y$ if and only if $x=y-w$ for some $w \in X$.
(a9) $x \leq y$ implies $x-z \leq y-z$ and $z-y \leq z-x$ for all $z \in X$.
(a10) $x, y \leq z$ implies $x-y=x \wedge(z-y)$.
(a11) $(x \wedge y)-(x \wedge z) \leq x \wedge(y-z)$.
(a12) $(x-y)-z=(x-z)-(y-z)$.

As a weak form of a subtraction algebra, Jun et al. discussed the weak subtraction algebras as follows:

Definition $2.1([8])$. By a weak subtraction algebra (WS-algebra), we mean a triplet $(W,-, 0)$, where $W$ is a nonempty set, - is a binary operation on $W$ and $0 \in W$ is a nullary operation, called zero element, such that

(S3) $(\forall x, y, z \in W)((x-y)-z=(x-z)-y)$,

(S4) $(\forall x \in W)(x-0=x, x-x=0)$,

(a12) $(\forall x, y, z \in W)((x-y)-z=(x-z)-(y-z))$.

Note that every subtraction algebra is a WS-algebra, but the converse is not true in general (see [8]).

\section{Order systems and ideals in WS-algebras}

In what follows, let $X$ denote a WS-algebra unless otherwise specified.

Definition 3.1. A nonempty subset $A$ of $X$ is called an ideal of $X$ if it satisfies (b1) $0 \in A$ 
(b2) $(\forall x \in X)(\forall y \in A)(x-y \in A \Rightarrow x \in A)$.

The set of all ideals of $X$ will be denoted by $\operatorname{Id}(X)$.

Lemma 3.2. An ideal $A$ of a subtraction algebra $X$ has the following property:

$$
(\forall x \in X)(\forall y \in A)(x \leq y \Rightarrow x \in A) .
$$

Proof. Straightforward.

Theorem 3.3. Let $A$ be a nonempty subset of $X$. Then the set

$$
K:=\left\{\begin{array}{l|l}
x \in X & \begin{array}{l}
\left(\cdots\left(\left(x-a_{1}\right)-a_{2}\right)-\cdots\right)-a_{n}=0 \\
\text { for some } a_{1}, a_{2}, \ldots, a_{n} \in A
\end{array}
\end{array}\right\}
$$

is a minimal ideal of $X$ containing $A$.

Proof. It is similar to the proof of Theorem 3.2 in [6].

We say that the ideal $K$ is the ideal generated by $A$, and is denoted by $\langle A\rangle$.

Definition 3.4. An ideal $A$ of $X$ is said to be irreducible if for any ideals $C$ and $D$ of $X, A=C \cap D$ implies $A=C$ or $A=D$.

Theorem 3.5. If $A \in \operatorname{Id}(X)$ satisfies the following assertion:

$$
(\forall x, y \in X \backslash A)(\exists z \in X \backslash A)(z-x \in A, z-y \in A),
$$

then $A$ is an irreducible ideal of $X$.

Proof. Assume that $A \in I d(X)$ satisfies (1). Let $C, D \in I d(X)$ be such that $A=C \cap D, A \neq C$ and $A \neq D$. Then there exist $x \in C \backslash A \subset X \backslash A$ and $y \in D \backslash A \subset X \backslash A$. It follows from (1) that there exists $z \in X \backslash A$ such that $z-x \in A$ and $z-y \in A$. Since $x \in C$ and $z-x \in A=C \cap D \subset C$, we have $z \in C$ because $C$ is an ideal of $X$. Also, $y \in D$ and $z-y \in D$, which imply $z \in D$. Hence $z \in C \cap D=A$, which is a contradiction. Hence $A$ is an irreducible ideal of $X$.

Corollary 3.6 ([11]). Let $A \in I d(X)$. Assume that for any $x, y \in X \backslash A$, there exists $z \in X \backslash A$ such that $z \leq x$ and $z \leq y$. Then $A$ is an irreducible ideal of $X$.

Definition 3.7. Let $X$ be a poset. A nonempty subset $I$ of $X$ is called an order system of $X$ if it satisfies:

(b3) $(\forall x \in X)(\forall y \in I)(x \leq y \Rightarrow x \in I)$,

(b4) $(\forall x, y \in I)(\exists z \in I)(x \leq z, y \leq z)$.

The set of all order systems of a poset $X$ will be denoted by $O_{s}(X)$. Note that if $X$ is a poset with the bottom element $\perp$, then every order system of $X$ contains the bottom element $\perp$.

Example 3.8. Let $X=\{0, a, b, c, d\}$ be a poset with the following Hasse diagram: 


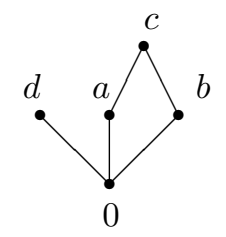

Then $I_{1}:=\{0, a\} \in O_{s}(X), I_{2}:=\{0, a, b, c\} \in O_{s}(X)$, but $J_{1}:=\{0, b, c\} \notin$ $O_{s}(X)$ and $J_{2}:=\{0, a, d\} \notin O_{s}(X)$.

Theorem 3.9. For every WS-algebra $X$, we have $O_{s}(X) \subset I d(X)$.

Proof. Let $I \in O_{s}(X)$. Since $I$ is nonempty, obviously $0 \in I$. Now let $x, y \in X$ satisfy $x-y \in I$ and $y \in I$. Then there exists $z \in I$ such that $x-y \leq z$ and $y \leq z$ by (b4). It follows from (a2) and (a12) that

$$
x-z=(x-z)-0=(x-z)-(y-z)=(x-y)-z=0 \in I
$$

so from (b2) that $x \in I$. Therefore $I \in I d(X)$, and so $O_{s}(X) \subset I d(X)$.

The following example shows that an ideal is not an order system in general.

Example 3.10. (1) Let $X=\{0, a, b, c, d\}$ be a set with the following Cayley table:

\begin{tabular}{c|ccccc}
- & 0 & $a$ & $b$ & $c$ & $d$ \\
\hline 0 & 0 & 0 & 0 & 0 & 0 \\
$a$ & $a$ & 0 & $a$ & 0 & $a$ \\
$b$ & $b$ & $b$ & 0 & 0 & $b$ \\
$c$ & $c$ & $b$ & $a$ & 0 & $c$ \\
$d$ & $d$ & $d$ & $d$ & $d$ & 0
\end{tabular}

Then $(X,-)$ is a subtraction algebra, and hence a WS-algebra. It is easy to verify that $Q_{1}:=\{0, a, d\} \in I d(X)$, but $Q_{1}:=\{0, a, d\} \notin O_{s}(X)$.

(2) Let $X=\{0, a, b, c, d\}$ be a set with the following Cayley table:

\begin{tabular}{c|ccccc}
- & 0 & $a$ & $b$ & $c$ & $d$ \\
\hline 0 & 0 & 0 & 0 & 0 & 0 \\
$a$ & $a$ & 0 & 0 & $a$ & 0 \\
$b$ & $b$ & $b$ & 0 & $b$ & $b$ \\
$c$ & $c$ & $c$ & $c$ & 0 & $c$ \\
$d$ & $d$ & $d$ & $d$ & $d$ & 0
\end{tabular}

Then $(X,-)$ is a WS-algebra, which is not a subtraction algebra. It is easy to verify that $Q_{2}:=\{0, a, c\} \in I d(X)$, but $Q_{2}:=\{0, a, c\} \notin O_{s}(X)$.

To make an ideal to be an order system, we need more strong condition.

Definition 3.11 ([9]). A subtraction algebra $X$ is said to be complicated if for any $a, b \in X$ the set

$$
\mathscr{G}(a, b):=\{x \in X \mid x-a \leq b\}
$$

has the greatest element. 
The greatest element of $\mathscr{G}(a, b)$ is denoted by $a+b$.

Lemma $3.12([9])$. If $X$ is a complicated subtraction algebra, then

$$
(\forall a, b \in X)(a \leq a+b, b \leq a+b) .
$$

Theorem 3.13. In a complicated subtraction algebra $X$, every ideal is an order system.

Proof. Let $Q$ be an ideal of a complicated subtraction algebra $X$. The condition (b3) follows from Lemma 3.2. Now let $x, y \in Q$. Since $(x+y)-x \leq y$, it follows from Lemma 3.2 and (b2) that $x+y \in Q$ so from Lemma 3.12 that (b4) is valid. Hence $Q$ is an order system of $X$.

Corollary 3.14 ([9]). Let $Q$ be a nonempty subset of a complicated subtraction algebra $X$. Then $Q$ is an ideal of $X$ if and only if $Q$ is an order system of $X$.

Theorem 3.15. Let $Q \in O_{s}(X)$. If $Q$ is irreducible as an ideal of $X$, then

$$
(\forall a, b \in X \backslash Q)(\exists c \in X \backslash Q)(c \leq a, c \leq b) \text {. }
$$

Proof. Assume that

$$
(\exists a, b \in X \backslash Q)(\forall c \in X)(c \leq a, c \leq b \Rightarrow c \in Q) .
$$

Let $Q(a)$ and $Q(b)$ be the ideals of $X$ generated by $Q \cup\{a\}$ and $Q \cup\{b\}$ respectively. Then $Q \subset Q(a) \cap Q(b)$. Let $x \in Q(a) \cap Q(b)$. Then $x \in Q(a)$ and $x \in Q(b)$. Thus

$$
\left(\cdots\left(\left((x-a)-c_{1}\right)-c_{2}\right)-\cdots\right)-c_{m}=0
$$

and

$$
\left(\cdots\left(\left((x-b)-d_{1}\right)-d_{2}\right)-\cdots\right)-d_{n}=0
$$

for some $c_{1}, c_{2}, \ldots, c_{m}, d_{1}, d_{2}, \ldots, d_{n} \in Q$. Since $Q$ is an ideal of $X$, it follows from (b1) and (b2) that $x-a \in Q$ and $x-b \in Q$ so from (b4) that there exists $z \in Q$ such that $x-a \leq z$ and $x-b \leq z$. Hence

$$
(x-z)-a=(x-a)-z=0 \text { and }(x-z)-b=(x-b)-z=0,
$$

and so $x-z \in Q$ by (2). But $Q \in I d(X)$ and $z \in Q$, and thus $x \in Q$ by (b2). Thus $Q(a) \cap Q(b) \subset Q$, and consequently $Q=Q(a) \cap Q(b)$ which is a contradiction.

\section{Right fixed maps}

Definition 4.1. A right fixed map $\alpha$ of $X$ is defined to be a self map $\alpha: X \rightarrow X$ satisfying $\alpha(x-y)=\alpha(x)-y$ for all $x, y \in X$.

Example 4.2. (1) Let $X=\{0, a, b\}$ be a set with the following Cayley table:

\begin{tabular}{c|ccc}
- & 0 & $a$ & $b$ \\
\hline 0 & 0 & 0 & 0 \\
$a$ & $a$ & 0 & $a$ \\
$b$ & $b$ & $b$ & 0
\end{tabular}


Then $(X,-)$ is a subtraction algebra, and hence a WS-algebra. It can be easily verify that the self map $\alpha$ of $X$ defined by $\alpha(0)=0, \alpha(a)=0$, and $\alpha(b)=b$ is a right fixed map.

(2) Consider a subtraction algebra, and hence a WS algebra, $X=\{0, a, b, c\}$ with the following Cayley table:

\begin{tabular}{c|cccc}
- & 0 & $a$ & $b$ & $c$ \\
\hline 0 & 0 & 0 & 0 & 0 \\
$a$ & $a$ & 0 & $a$ & $a$ \\
$b$ & $b$ & $b$ & 0 & $b$ \\
$c$ & $c$ & $c$ & $c$ & 0
\end{tabular}

Let $\beta: X \rightarrow X$ be defined by $\beta(0)=0, \beta(a)=0, \beta(b)=c$, and $\beta(c)=c$. Then $\beta$ is not a right fixed map since $\beta(b-c) \neq \beta(b)-c$.

(3) Let $X=\{0, a, b, c, d\}$ be a set with the following Cayley table:

\begin{tabular}{c|ccccc}
- & 0 & $a$ & $b$ & $c$ & $d$ \\
\hline 0 & 0 & 0 & 0 & 0 & 0 \\
$a$ & $a$ & 0 & 0 & 0 & 0 \\
$b$ & $b$ & $b$ & 0 & $b$ & $b$ \\
$c$ & $c$ & $c$ & $c$ & 0 & $c$ \\
$d$ & $d$ & $d$ & $d$ & $d$ & 0
\end{tabular}

Then $(X,-)$ is a WS-algebra, which is not a subtraction algebra. Let $\gamma$ be a self map of $X$ defined by $\gamma(0)=\gamma(a)=\gamma(b)=0, \gamma(c)=c$ and $\gamma(d)=d$. Then $\gamma$ is a right fixed map.

(4) Let $X=\{0, a, b, c, d\}$ be a set with the following Cayley table:

\begin{tabular}{c|ccccc}
- & 0 & $a$ & $b$ & $c$ & $d$ \\
\hline 0 & 0 & 0 & 0 & 0 & 0 \\
$a$ & $a$ & 0 & 0 & 0 & 0 \\
$b$ & $b$ & $b$ & 0 & 0 & $b$ \\
$c$ & $c$ & $c$ & $c$ & 0 & $c$ \\
$d$ & $d$ & $d$ & $d$ & $d$ & 0
\end{tabular}

Then $(X,-)$ is a WS-algebra, which is not a subtraction algebra. Let $\alpha$ be a self map of $X$ defined by

$$
\alpha(x)= \begin{cases}0 & \text { if } x \in\{0, a\} \\ x & \text { otherwise }\end{cases}
$$

Then $\alpha$ is a right fixed map of $X$.

Proposition 4.3. If $\alpha$ is a right fixed map of $X$, then

(i) $\alpha(0)=0$,

(ii) $(\forall x \in X)(\alpha(0-x)=0)$,

(iii) $(\forall x \in X)(\alpha(x) \leq x)$,

(iv) $(\forall x, y \in X)(x \leq y \Rightarrow \alpha(x) \leq y)$. 
Proof. (i) For every $x, y \in X$, we have

$$
\alpha(0)=\alpha(0-\alpha(0))=\alpha(0)-\alpha(0)=0 .
$$

(ii) For every $x \in X$, we have $\alpha(0-x)=\alpha(0)=0$.

(iii) For any $x \in X$, we get $0=\alpha(0)=\alpha(x-x)=\alpha(x)-x$, and so $\alpha(x) \leq x$.

(iv) Assume that $x \leq y$ for every $x, y \in X$. Then $0=\alpha(0)=\alpha(x-y)=$ $\alpha(x)-y$, and so $\alpha(x) \leq y$.

Definition 4.4. For a right fixed map $\alpha$ of $X$, the kernel of $\alpha$, denoted by $\operatorname{ker}(\alpha)$, is defined to be the set

$$
\operatorname{ker}(\alpha)=\{x \in X \mid \alpha(x)=0\} .
$$

Obviously $\operatorname{ker}(\alpha) \neq \emptyset$ since $0 \in \operatorname{ker}(\alpha)$.

Theorem 4.5. Let $\alpha$ be a right fixed map of $X$. Then $\alpha$ is one-to-one if and only if $\operatorname{ker}(\alpha)=0$.

Proof. Assume that $\alpha$ is one-to-one and let $x \in \operatorname{ker}(\alpha)$. Then $\alpha(x)=0=\alpha(0)$, and thus $x=0$, i.e., $\operatorname{ker}(\alpha)=\{0\}$. Conversely suppose that $\operatorname{ker}(\alpha)=\{0\}$. Let $x, y \in X$ satisfy $\alpha(x)=\alpha(y)$. Since $\alpha(y) \leq y$, it follows from (a9) that $\alpha(x-y)=\alpha(x)-y \leq \alpha(x)-\alpha(y)=0$ so that $\alpha(x-y)=0$. Hence $x-y \in \operatorname{ker}(\alpha)$, and so $x-y=0$. Similarly, $y-x=0$. This proves that $x=y$. Therefore $\alpha$ is one-to-one.

Theorem 4.6. Let $\alpha$ be a right fixed map of $X$. Then $\alpha$ is one-to-one if and only if $\alpha$ is the identity map.

Proof. Sufficiency is obvious. Suppose that $\alpha$ is one-to-one. For every $x \in X$, we have

$$
\alpha(x-\alpha(x))=\alpha(x)-\alpha(x)=0=\alpha(0)
$$

and so $x-\alpha(x)=0$, i.e., $x \leq \alpha(x)$. Since $\alpha(x) \leq x$ for all $x \in X$, it follows that $\alpha(x)=x$ so that $\alpha$ is the identity map.

Theorem 4.7. Let $\alpha$ be a right fixed map of $X$. If $\alpha$ is idempotent, i.e., $\alpha(\alpha(x))=\alpha(x)$ for all $x \in X$, then

(i) $(\forall x \in X)(\alpha(x)=x \Leftrightarrow x \in \operatorname{Im}(\alpha))$.

(ii) $\operatorname{ker}(\alpha) \cap \operatorname{Im}(\alpha)=\{0\}$.

Proof. (i) Necessity is obvious. If $x \in \operatorname{Im}(\alpha)$, then $\alpha(y)=x$ for some $y \in X$. Thus $\alpha(x)=\alpha(\alpha(y))=\alpha(y)=x$.

(ii) If $x \in \operatorname{ker}(\alpha) \cap \operatorname{Im}(\alpha)$, then $\alpha(x)=0$ and $\alpha(y)=x$ for some $y \in X$. It follows that

so that $\operatorname{ker}(\alpha) \cap \operatorname{Im}(\alpha)=\{0\}$.

$$
0=\alpha(x)=\alpha(\alpha(y))=\alpha(y)=x
$$

The following example shows that a WS-algebra $X$ does not satisfy the assertion (a8) in general. 
Example 4.8. Let $X=\{0, a, b, c, d\}$ be a WS-algebra, which is not a subtraction algebra, described in Example 4.2(4). We know that $b \leq c$, but there does not exist $w \in X$ such that $b=c-w$.

Theorem 4.9. Let $\alpha$ be a right fixed map of a subtraction algebra $X$. Then

(i) $(\forall x \in X)(\exists y \in \operatorname{ker}(\alpha), \exists z \in \operatorname{Im}(\alpha))(z=x-y)$.

(ii) $\alpha$ is idempotent.

Proof. Since $\alpha(x) \leq x$ for all $x \in X$, it follows from (a8) that $\exists w \in X$ such that $\alpha(x)=x-w$ so from (a6) that

$$
x-(x-\alpha(x))=x-(x-(x-w))=x-w=\alpha(x) .
$$

Noticing that $x-\alpha(x) \in \operatorname{ker}(\alpha)$ and $\alpha(x) \in \operatorname{Im}(\alpha)$, we have the result (i). Moreover, using (a1) implies that

$$
\alpha(\alpha(x))=\alpha(x-w)=\alpha(x)-w=(x-w)-w=x-w=\alpha(x)
$$

for all $x \in X$, which proves (ii).

Corollary 4.10. If $\alpha$ is a right fixed map of a subtraction algebra $X$, then

(i) $(\forall x \in X)(\alpha(x)=x \Leftrightarrow x \in \operatorname{Im}(\alpha))$.

(ii) $\operatorname{ker}(\alpha) \cap \operatorname{Im}(\alpha)=\{0\}$.

Denote by $R F(X)$ the set of all right fixed maps of $X$. Let $\ominus$ be a binary operation on $R F(X)$ defined by $(\alpha \ominus \beta)(x)=\alpha(x)-\beta(x)$ for all $\alpha, \beta \in R F(X)$ and $x \in X$. It is easy to verify that if $X$ is a WS-algebra, then $(R F(X), \ominus)$ is a WS-algebra. Let $\operatorname{IRF}(X)$ denote the set of all idempotent right fixed maps of $X$.

Theorem 4.11. For every $\alpha, \beta \in I R F(X)$, if $\alpha \ominus \beta=0$ in $R F(X)$, then $\operatorname{Im}(\alpha) \subset \operatorname{Im}(\beta)$.

Proof. Let $\alpha, \beta \in I R F(X)$ satisfy $\alpha \ominus \beta=0$. If $y \in \operatorname{Im}(\alpha)$, then $\alpha(y)=y$ by Theorem 4.7, and hence

$$
0=(\alpha \ominus \beta)(y)=\alpha(y)-\beta(y)=y-\beta(y),
$$

i.e., $y \leq \beta(y)$. Combining this with Proposition 4.3(iii), we get $y=\beta(y) \in$ $\operatorname{Im}(\beta)$. Hence $\operatorname{Im}(\alpha) \subset \operatorname{Im}(\beta)$.

Theorem 4.12. Let $\alpha, \beta \in I R F(X)$. Then

(i) $\alpha \ominus \beta \in R F(X)$.

(ii) If $\alpha(\beta(x))=\beta(\alpha(x))$ for all $x \in X$, then $\alpha \ominus \beta \in \operatorname{IRF}(X)$.

(iii) If $\operatorname{Im}(\alpha) \subset \operatorname{Im}(\beta)$ and $\alpha(\beta(x))=\beta(\alpha(x))$ for all $x \in X$, then $\alpha \ominus \beta=0$ in $R F(X)$.

(iv) $\operatorname{Im}(\alpha) \cap \operatorname{ker}(\beta) \subset \operatorname{Im}(\alpha \ominus \beta)$. 
Proof. (i) For every $x, y \in X$, we have

$$
\begin{aligned}
(\alpha \ominus \beta)(x-y) & =\alpha(x-y)-\beta(x-y) \\
& =(\alpha(x)-y)-(\beta(x)-y) \\
& =(\alpha(x)-\beta(x))-y \\
& =(\alpha \ominus \beta)(x)-y,
\end{aligned}
$$

and so $\alpha \ominus \beta \in R F(X)$.

(ii) Assume that $\alpha(\beta(x))=\beta(\alpha(x))$ for all $x \in X$. Let $x \in X$. Then

$$
\begin{aligned}
(\alpha \ominus \beta)((\alpha \ominus \beta)(x)) & =(\alpha \ominus \beta)(\alpha(x)-\beta(x)) \\
& =\alpha(\alpha(x)-\beta(x))-\beta(\alpha(x)-\beta(x)) \\
& =(\alpha(\alpha(x))-\beta(x))-(\beta(\alpha(x))-\beta(x)) \\
& =(\alpha(x)-\beta(x))-(\alpha(\beta(x))-\beta(x)) \\
& =(\alpha(x)-\beta(x))-\alpha(\beta(x)-\beta(x)) \\
& =(\alpha(x)-\beta(x))-\alpha(0) \\
& =\alpha(x)-\beta(x) \\
& =(\alpha \ominus \beta)(x),
\end{aligned}
$$

that is, $\alpha \ominus \beta$ is idempotent. Hence $\alpha \ominus \beta \in I R F(X)$.

(iii) Suppose that $\operatorname{Im}(\alpha) \subset \operatorname{Im}(\beta)$ and $\alpha(\beta(x))=\beta(\alpha(x))$ for all $x \in X$. Since $\alpha(x) \in \operatorname{Im}(\alpha) \subset \operatorname{Im}(\beta)$ for all $x \in X$, it follows from Theorem 4.7 that

$$
\begin{aligned}
(\alpha \ominus \beta)(x) & =\alpha(x)-\beta(x)=\beta(\alpha(x))-\beta(x) \\
& =\alpha(\beta(x))-\beta(x)=\alpha(\beta(x)-\beta(x)) \\
& =\alpha(0)=0
\end{aligned}
$$

for all $x \in X$. Therefore $\alpha \ominus \beta=0$.

(iv) If $y \in \operatorname{Im}(\alpha) \cap \operatorname{ker}(\beta)$, then $\beta(y)=0$ and $\alpha(x)=y$ for some $x \in X$. It follows from (a2) that

$$
y=\alpha(x)=\alpha(\alpha(x))-0=\alpha(y)-\beta(y)=(\alpha \ominus \beta)(y) \in \operatorname{Im}(\alpha \ominus \beta) .
$$

Therefore $\operatorname{Im}(\alpha) \cap \operatorname{ker}(\beta) \subset \operatorname{Im}(\alpha \ominus \beta)$.

We pose a problem: If $\alpha \in R F(X)$, then is $\operatorname{ker}(\alpha)$ an order system (or, an ideal) of $X$ ?

\section{References}

[1] J. C. Abbott, Semi-Boolean algebras, Matemat. Vesnik 4 (1967), 177-198.

[2] _ Sets, Lattices and Boolean Algebras, Allyn and Bacon, Boston 1969.

[3] S. S. Ahn, Y. H. Kim, and K. J. Lee, A relation on subtraction algebras, Sci. Math. Jpn. Online e-2005 (2005), 51-55.

[4] G. Birkhoff, Lattice Theory, Amer. Math. Soc. Colloq. Publ., Vol. 25, second edition 1984; third edition, 1967, Providence.

[5] G. Grätzer, Universal Algebra, 2nd edition, Springer-Verlag, New York Inc., 1979.

[6] Y. B. Jun and H. S. Kim, On ideals in subtraction algebras, Sci. Math. Jpn. Online e-2006 (2006), 1081-1086. 
[7] Y. B. Jun, H. S. Kim, and K. J. Lee, The essence of subtraction algebras, Sci. Math. Jpn. Online e-2006 (2006), 1069-1074.

[8] Y. B. Jun, Y. H. Kim, and K. J. Lee, Weak forms of subtraction algebras, Bull. Korean Math. Soc. (submitted).

[9] Y. B. Jun, Y. H. Kim, and K. A. Oh, Subtraction algebras with additional conditions, Commun. Korean Math. Soc. (submitted).

[10] Y. B. Jun, H. S. Kim, and E. H. Roh, Ideal theory of subtraction algebras, Sci. Math. Jpn. Online e-2004 (2004), 397-402.

[11] Y. B. Jun and K. H. Kim, Prime and irreducible ideals in subtraction algebras, Ital. J. Pure Appl. Math. (submitted)

[12] Y. B. Jun, X. L. Xin, and E. H. Roh, A class of algebras related to BCI-algebras and semigroups, Soochow J. Math. 24 (1998), no. 4, 309-321.

[13] Y. H. Kim and H. S. Kim, Subtraction algebras and BCK-algebras, Math. Bohemica 128 (2003), no. 1, 21-24.

[14] B. M. Schein, Difference Semigroups, Comm. Algebra 20 (1992), 2153-2169.

[15] B. Zelinka, Subtraction Semigroups, Math. Bohemica 120 (1995), 445-447.

Young Bae Jun

Department of Mathematics Education (and RINS)

Gyeongsang National University

Chinju 660-701, Korea

E-mail address: skywine@gmail.com

Chul Hwan Park

Department of Mathematics

UNIVERSITY OF ULSAN

ULSAN 680-749, KOREA

E-mail address: chpark@ulsan.ac.kr or skyrosemary@gmail.com

Eun HWan RoH

Department of Mathematics Education

Chinju National University of Education

Chinju 660-756, Korea

E-mail address: ehroh@cue.ac.kr 